J. NARITA

KODAI MATH. I.

13 (1990). 311-316

\title{
INTERPOLATING SEQUENCES ON PLANE DOMAINS
}

\author{
Dedicated to Professor T. Fuji'i'e on his 60th birthday
}

\section{BY JUNICHIRO NARITA}

1. Let $D$ be an arbitrary open set in $C$, and $H^{\infty}(D)$ be the set of all bounded analytic functions on $D$. $A$ sequence $\left\{z_{j}\right\}$ in $D$ is called $\left(H^{\infty}(D)\right.$-) interpolating sequence if for all bounded sequences $\left\{a_{j}\right\}$, the interpolation problem

$$
f\left(z_{j}\right)=a_{\jmath}, \quad j=1,2,3, \cdots
$$

has a solution $f(z)$ in $H^{\infty}(D)$. Since $\mathrm{L}$. Carleson characterized the interpolating sequence in the unit disk in [3], several authors have studied interpolating sequences in more general plane domains (for example [2], [7]). In this paper we study interpolating sequences in an arbitrary plane domain and in a certain plane domain satisfying some geometrical condition.

In $\S 2$, we show the following theorem by which we see that the interpolating sequence can be characterized in terms of its local behavior.

THEOREM 1. Let $S=\left\{z_{j}\right\}$ be a sequence in an arbitrary open set $D$ of the complex plane $\boldsymbol{C}$. If for all $\zeta \in C$, there exists some neighborhood $U$ of $\zeta$ such that $S \cap U$ is an $H^{\infty}(D \cap U)$-interpolating sequence, then $S$ is an $H^{\infty}(D)$-interpolating sequence.

When $\left\{z_{j}\right\}$ is an $H^{\infty}(D)$-interpolating sequence, the open mapping theorem shows that

$$
M=\sup _{\|\left(a_{j} \|_{\infty} \leq 1\right.} \inf \left\{\|f\|_{\infty}: f \in H^{\infty}(D), f\left(z_{\jmath}\right)=a_{\jmath}, \quad j=1,2,3, \cdots\right\}<\infty,
$$

where $\left\|\left\{a_{j}\right\}\right\|_{\infty}=\sup \left|a_{j}\right|$. This constant $M$ is called the constant of interpolation for $\left\{z_{j}\right\}$ (in $D$ ). For $\Delta=\{|z|<1\}$, L. Carleson [3] showed that a sequence $\left\{z_{j}\right\}$ in $\Delta$ is the interpolating sequence if and only if

$$
\delta=\inf _{k} \prod_{\substack{j=1 \\ j \neq k}}^{\infty}\left|\frac{z_{k}-z_{j}}{1-\bar{z}_{k} z_{j}}\right|>0 .
$$

The constant of interpolation $M$ for $\left\{z_{j}\right\}$ is estimated by the inequalities (see

Received May 8, 1989; revised Decembea 22, 1989. 
[6] p. 287)

$$
\frac{1}{\delta} \leqq M \leqq \frac{c}{\delta}\left(1+\log \frac{1}{\delta}\right),
$$

where $c$ is the absolute constant.

By use of Theorem 1, we generalize the result of L. Carleson for some class of plane domains, and we also study the constant of interpolation for these domains. To this purpose, we introduce a metric $\rho$ for a plane domain $D$ such that $C \backslash D$ consists of at least two points. In this case the universal covering surface of $D$ is the open unit disk $\Delta$. Let $\pi: \Delta \rightarrow D$ be the universal covering map, and let $\lambda_{\Delta}$ be the Poincaré (or hyperbolic) distance in $\Delta$. The Poincaré distance $\lambda$ for $D$ is defined as

$$
\lambda(a, b)=\inf \left\{\lambda_{\Delta}(c, d) ; \pi(c)=a, \pi(d)=b\right\} .
$$

(cf. [9] p. 43). We set $\rho=\left(e^{\lambda}-1\right) /\left(e^{\lambda}+1\right)$. For $D=\{|z|<1\}, \rho$ is exactly the pseudo-hyperbolic distance $\rho(a, b)=|(a-b) /(1-\bar{a} b)|$ and $\lambda=\lambda_{\Delta}=\log |(1+\rho) /(1-\rho)|$.

THEOREM 2. Let $D$ be a domain in $C$. Suppose the Euclidean diameter of $D$ is less than $R(<\infty)$, and the diameter of every component of $C \backslash D$ exceeds some positive constant $\varepsilon$. Then the sequence $\left\{z_{j}\right\}$ in $D$ is interpolating sequence if and only if

$$
\delta=\inf _{\substack { k \\
\begin{subarray}{c}{j=1 \\
j \neq k{ k \\
\begin{subarray} { c } { j = 1 \\
j \neq k } }\end{subarray}}^{\infty} \rho\left(z_{\jmath}, z_{k}\right)>0 .
$$

Furthermore, the constant of interpolation $M$ for $\left\{z_{j}\right\}$ is bounded above by the constant which depends only on the ratio $R / \varepsilon$ and $\delta$.

2. To prove theorem 1 we use the Vitushkin operator

$$
\left(T_{g} f\right)(w)=\frac{1}{\pi} \iint_{C} \frac{f(z)-f(w)}{z-w} \frac{\partial g}{\partial \bar{z}} d x d y, \quad z=x+i y, w \in \boldsymbol{C},
$$

which is defined for a bounded Borel function $f$ and a $C^{1}$-function $g$ with compact support.

Lemma. Let $D$ be an open set in $C, \zeta \in C$, and $U=\{|z-\zeta|<c\}$. Let $S$ be an $H^{\infty}(D \cap U)$-interpolating sequence with constant of interpolation $M$. Then for $V=\{|z-\zeta|<c / 136 M\}, S \cap V$ is an $H^{\infty}(D)$-interpolating sequence whose constant of interpolation is less than $66 \mathrm{M}$.

Proof. Let $S \cap V=\left\{z_{j}\right\}$, and let $\left\{c_{j}\right\}$ be a bounded sequence with $\left\|\left\{c_{j}\right\}\right\|_{\infty} \leqq 1$. Then there exists $f_{1} \in H^{\infty}(D \cap U)$ such that

$$
f_{1}\left(z_{j}\right)=c_{j}(j=1,2, \cdots), \quad\left\|f_{1}\right\| \leqq M .
$$


We can take a $C^{1}$-function $g$ with a compact supprot in $U$, which satisfies $g=1$ on $\{|z-\zeta|<c / 2\}$, and $\|\partial g / \partial \bar{z}\|_{\infty} \leqq 4 / c$. We extend the domain of definition of $f_{1}$ by setting $f_{1}=0$ to the outside of $D \cap U$. Then by the property of Vitushkin operator (see [5] p. 4-5), $f_{1}-T_{g} f_{1}$ is analytic on $\{|z-\zeta|<c / 2\}$,

is analytic on $D$, and

$$
F_{1}=T_{g} f_{1}-\left(T_{g} f_{1}-f_{1}\right)(\zeta)
$$

$$
\begin{aligned}
\left\|T_{g} f_{1}\right\|_{\infty} & \leqq \frac{2}{\pi}\left\|\frac{\partial g}{\partial \bar{z}}\right\|_{\infty} \operatorname{osc}(f, \operatorname{supp}(g))[\pi \text { Area }(\operatorname{supp}(g))]^{1 / 2} \\
& \leqq \frac{2}{\pi} \cdot \frac{4}{c} \cdot 2\left\|f_{1}\right\|_{\infty} \pi c \leqq 16 M .
\end{aligned}
$$

Hence $\left\|F_{1}\right\|_{\infty} \leqq 33 M$, and $\left\|F_{1}-f_{1}\right\|_{\infty} \leqq 34 M$. Since

$$
F_{1}-f_{1}=T_{g} f_{1}-f_{1}-\left(T_{g} f_{1}-f_{1}\right)(\zeta)
$$

is analytic on $\{|z-\zeta|<c / 2\}$ and equals 0 at $\zeta$, by the lemma of Schwarz, we see that on $V$,

$$
\left|F_{1}-f_{1}\right| \leqq\left\|F_{1}-f_{1}\right\|_{\infty} \frac{c / 136 M}{c / 2} \leqq \frac{1}{2} .
$$

For the sequence $\left\{\left(f_{1}-F_{1}\right)\left(z_{j}\right)\right\}$, we can take $f_{2} \in H^{\infty}(D \cap U)$ as

$$
\begin{aligned}
& f_{2}\left(z_{j}\right)=\left(f_{1}-F_{1}\right)\left(z_{j}\right) \quad(j=1,2, \cdots), \\
& \left\|f_{2}\right\| \leqq M\left\|\left\{\left(f_{1}-F_{1}\right)\left(z_{j}\right)\right\}\right\|_{\infty} \leqq \frac{M}{2} .
\end{aligned}
$$

By repeating this process, we get two sequences $\left\{f_{n}\right\}$ of $H^{\infty}(D \cap U)$-functions and $\left\{F_{n}\right\}$ of $H^{\infty}(D)$-functions such that

$$
\left(F_{1}+F_{2}+\cdots+F_{n}+f_{n+1}\right)\left(z_{j}\right)=c_{\jmath},
$$

$\left\|f_{n+1}\right\| \leqq M / 2^{n}$, and $\left\|F_{n}\right\| \leqq 33\left\|f_{n}\right\|$.

Now $F=\sum_{n=1}^{\infty} F_{n}$ satisfies the interpolating condition $F\left(z_{j}\right)=c_{\jmath}(j=1,2, \cdots)$ and

$$
\|F\| \leqq \Sigma\left\|F_{n}\right\| \leqq \Sigma \frac{33 M}{2^{n-1}}=66 M
$$

Let $X$ be a subset of the maximal ideal space $\mu(D)$ of $H^{\infty}(D)$. Then hull $(X)$ is defined by

$$
\begin{aligned}
& I_{X}=\left\{f \in H^{\infty}(D) ; \hat{f}=0 \text { on } X\right\}, \\
& \operatorname{hull}(X)=\left\{x \in \mathscr{M}(D) ; \hat{f}(x)=0 \text { for all } f \in I_{X}\right\},
\end{aligned}
$$

where $\hat{f}$ means the Gel'fand transformation of $f$. It is known that a sequence $S$ in $D$ is an interpolating sequence if and only if $S$ satisfies the conditions 
(i) $S$ is discrete,

(ii) $\bar{S}$ is homeomorphic to the Čech compactification of $S$,

(iii) $\operatorname{hull}(S)=\bar{S}$,

where the closure is taken in $\mathscr{M}(D)$. (see [8] p. 205).

Proof of Theorem 1. First we assume that the domain $D$ is bounded. For each $\zeta \in \boldsymbol{C}$, let $U_{\zeta}$ be a neighborhood of $\zeta$ which satisfies the assumption of the theorem. By the lemma, we can take a neighborhood $V_{\zeta}$ of $\zeta$, such that $S \cap V_{\zeta}$ is an $H^{\infty}(D)$-interpolating sequence. Then hull $\left(S \cap V_{\zeta}\right)=\overline{S \cap V_{\zeta}}$. Choose finitely many members $V_{\zeta_{1}}, \cdots, V_{\zeta_{m}}$ for which $\bigcup_{k=1}^{m} V_{\zeta_{k}} \supset \bar{D}$. Then

$$
\bar{S}=\overline{\bigcup_{k}\left(S \cap V_{\zeta_{k}}\right)}=\operatorname{hull}\left(\bigcup_{k}\left(S \cup V_{\zeta_{k}}\right)\right)=\operatorname{hull}(S) .
$$

Hence $S$ satisfies the above condition (iii). Conditions (i), (ii) are also derived from the fact that these are satisfied by $S \cap V_{\zeta_{k}}(k=1, \cdots, m)$. Now it is concluded that $S$ is an $H^{\infty}(D)$-interpolating sequence. One can apply the lemma also for $\zeta=\infty$ if one rotates $\hat{\boldsymbol{C}}(=\boldsymbol{C} \cup\{\infty\})$ by a suitable linear transformation. Therefore, the boundedness of $D$ is unnecessary.

In order to prove the uniform boundedness of constant of interpolation in Theorem 2, we shall need another version of Theorem 1. To state it, we introduce the Banach algebras $H^{\infty}\left(\left\{D_{n}\right\}\right)$. (cf. [1], [10]). Let $\left\{D_{n}\right\}_{n=1}^{\infty}$ be a sequence of open sets in $C$, all of which are contained in a bounded set. Let $\bigcup_{n=1}^{\infty}\left(D_{n} \times\{n\}\right)$ be the formal disjoint union of $\left\{D_{n}\right\}_{n=1}^{\infty}$. Let $H^{\infty}\left(\left\{D_{n}\right\}\right)$ be the Banach algebra of bounded analytic functions on $\bigcup_{n=1}^{\infty}\left(D_{n} \times\{n\}\right)$, and let $\mathscr{M}\left(\left\{D_{n}\right\}\right)$ be the maximal ideal space of this algebra. We can identify $\bigcup_{n=1}^{\infty}\left(D_{n} \times\{n\}\right)$ with a subset of $\mathscr{N}\left(\left\{D_{n}\right\}\right)$ by means of the usual point evaluations. Define a coordinate function $Z \in H^{\infty}\left(\left\{D_{n}\right\}\right)$ by $Z(\lambda, n)=\lambda$.

Theorem 1 is valid for these algebras as in the following form. Indeed, the proof is just the same if we exchange $U_{\zeta}, V_{\zeta}$ by $\hat{Z}^{-1}\left(U_{\zeta}\right), \hat{Z}^{-1}\left(V_{\zeta}\right)$ respectively.

THEOREM 1'. Let $\left\{D_{n}\right\}_{n=1}^{\infty}$ be as above, and let $S$ be a sequence in $\bigcup_{n=1}^{\infty}\left(D_{n} \times\right.$ $\{n\})$. If for all $\zeta \in C$, there exists some neighborhood $U$ of $\zeta$ such that $S \cap \hat{Z}^{-1}(U)$ is an $H^{\infty}\left(\left\{D_{n} \cap U\right\}\right)$-interpolating sequence, then $S$ is an $H^{\infty}\left(\left\{D_{n}\right\}\right)$-interpolating sequence.

3. Proof of Theorem 2. First, we assume that $\left\{z_{j}\right\}$ is an $H^{\infty}(D)$-interpolating sequence. Let $\Delta=\{|z|<1\}$, and let $\pi: \Delta \rightarrow D$ be a universal covering map. Let $M$ be a constant of interpolation of $\left\{z_{j}\right\}$. Then for any fixed $k$ we can take $f \in H^{\infty}(D)$ such that $\|f\| \leqq M, f\left(z_{k}\right)=1$, and $f\left(z_{j}\right)=0(j \neq k)$. Then $f \circ \pi \in$ 
$H^{\infty}(\Delta)$ and $\|f \circ \pi\| \leqq M$. We fix some $a_{k} \in \pi^{-1}\left(z_{k}\right)$, and we take each $a_{j} \in \pi^{-1}\left(z_{\jmath}\right)$ as satisfies

Therefore,

$$
\lambda_{\Delta}\left(a_{\jmath}, a_{k}\right)=\lambda_{D}\left(z_{\jmath}, z_{k}\right)
$$

$$
\rho_{D}\left(z_{\jmath}, z_{k}\right)=\rho_{\Delta}\left(z_{\jmath}, z_{k}\right)=\left|\frac{a_{j}-a_{k}}{1-\overline{a_{j}} a_{k}}\right|,
$$

where $\lambda_{\Delta}, \lambda_{D}$ and $\rho_{\Delta}, \rho_{D}$ are hyperbolic distance and pseudo hyperbolic distance for $\Delta, D$ respectively. Let $B$ be a Blaschke product with zeros $\left\{a_{j}\right\}_{j \neq k}$. There exists $g \in H^{\infty}(\Delta)$ such that $f \circ \pi=B g,\|g\| \leqq M$. By

$$
1=\left|f \circ \pi\left(a_{k}\right)\right|=\left|B\left(a_{k}\right)\right|\left|g\left(a_{k}\right)\right| \leqq M\left|B\left(a_{k}\right)\right|,
$$

we see that

$$
\prod_{\substack{j=1 \\ j \neq k}}^{\infty} \rho_{D}\left(z_{\jmath}, z_{k}\right)=\prod_{\substack{j=1 \\ j \neq k}}^{\infty}\left|\frac{a_{j}-a_{k}}{1-\overline{a_{j}} a_{k}}\right|=\left|B\left(a_{k}\right)\right| \geqq \frac{1}{M}>0 .
$$

As $M$ is not depend on $k$, we conclude that

$$
\inf _{k} \prod_{\substack{j=1 \\ j \neq k}}^{\infty} \rho_{D}\left(z_{\jmath}, z_{k}\right) \geqq \frac{1}{M}>0 .
$$

For the proof of the reverse implication, we use Theorem 1. For each $\zeta \in \boldsymbol{C}$, we take the neighborhood $U=\{z ;|z-\zeta|<\varepsilon / 2\}$. Then by the assumption on $D$, each component of $D \cap U$ is simply connected. In general, $D^{\prime} \subset D$ means $\lambda_{D^{\prime}} \geqq \lambda_{D}$. So we get

$$
\inf _{z_{k} \in D \cap U} \prod_{z_{\jmath}, z_{k} \in D_{\cap U, \jmath \neq k}} \rho_{D \cap U}\left(z_{\jmath}, z_{k}\right) \geqq \delta>0 .
$$

By Carleson's result concerning the unit disk, we see that $S \cap U$ is an $H^{\infty}(D \cap U)$ interpolating sequence. Then Theorem 1 implies that $S$ is an $H^{\infty}(D)$-interpolating sequence.

As to constant of interpolation, we use Theorem $1^{\prime}$ instead of Theorem 1 . Suppose there exist a sequence of open sets $\left\{R_{n}\right\}$, and a sequence of sequences $S_{n}=\left\{z_{n, j}\right\}_{j} \subset R_{n}$ all of which satisfies the condition of the theorem for the same $R$, $\varepsilon$, and $\delta$, and suppose the constants of interpolation $M_{n}$ are unbounded. This means that the sequence $S=\cup S_{n}$ is not an $H^{\infty}\left(\left\{R_{n}\right\}\right)$-interpolating sequence. By translating $R_{n}$ if necessary, we can assume all $R_{n}$ are contained in a bounded set in $C$. Then by use of Theorem $1^{\prime}$ we can conclude that $S$ is an $H^{\infty}\left(\left\{R_{n}\right\}\right)$-interpolating sequence. This is a contradiction.

REMARK. As the former part of the preceeding proof does not depend on the assumption on $D$ except that the universal covering surface of $D$ is the unit disk, it is always true that interpolating conditions implies inf $\Pi \rho\left(z_{\jmath}, z_{k}\right)$ $>0$. On the contrary, there exists the plane region $D$ and the sequence $\left\{z_{j}\right\}$ $\subset D$, for which $\Pi \rho\left(z_{\jmath}, z_{k}\right)>0$ but $\left\{z_{j}\right\}$ is not an interpolating sequence. 
We take $\Delta_{n}=\left\{z ;\left|z-c_{n}\right|<r_{n}\right\}, c_{n}>0$ as $\Delta_{n} \cap \Delta_{m}=\varnothing(n \neq m), \Delta_{n} \subset \Delta \backslash\{0\} \quad(n=$ $1,2,3, \cdots)$ and

$$
\sum_{n=1}^{\infty} \frac{r_{n}}{c_{n}}<\infty
$$

For $D=\Delta \backslash \bar{\cup} \Delta_{n}$, Zalcman [11] showed that for any $f \in H^{\infty}(D)$,

$$
\lim _{R^{-\ni} \rightarrow x \rightarrow 0} f(x)=\frac{1}{2 \pi i} \int_{\partial D} \frac{f(z)}{z} d z
$$

where $R^{-}=\{x \in \boldsymbol{R} ; x<0\}$. Hence any sequence $\left\{z_{j}\right\} \subset R^{-}$, tending to 0 , can not be an interpolating sequence. Nevertheless, by taking $z_{j} \rightarrow 0$ sufficiently fast, we can get the sequence $\left\{z_{j}\right\}$ which also satisfies inf $\Pi \rho\left(z_{\jmath}, z_{k}\right)>0$.

\section{REFERENCES}

[1] M. Behrens, The maximal ideal space of algebras of bounded analytic functions on infinitely connected domains, Trans. Amer. Math. Soc. 161 (1971), 358-380.

[2] M. Behrens, Interpolation and Gleason parts in L-domains, Trans. Amer. Math. Soc. 286 (1984), 203-225.

[3] L. CARLEson, An interpolation problem for bounded analytic functions, Amer. J. Math. 80 (1958), 921-930.

[4] T. Gamelin, Localization of the corona problem, Pacific J. Math. 34 (1970), 73-81.

[5] T. Gamelin, Lectures on $H^{\infty}(D)$, Univ. Nacional de la Plata, Argentine, 1972.

[6] J. Garnett, Bounded analytic functions, Academic Press, New York, 1981.

[7] J. GARnetT and P. Jones, The corona theorem for Denjoy domains, Acta Math. 155 (1985), 27-40.

[8] K. Hoffman, Banach spaces of analytic functions, Prentice-Hall, Englewood Cliffs, N. J., 1962.

[9] I. KRA, Automorphic forms and Kleinian groups, W. A. Benjamin, Reading, Massachusetts, 1972.

[10] J. NARITA, A remark on the corona problem for plane domains, J. Math. Kyoto Univ. 25 (1985), 293-298.

[11] L. ZALCMAN, Bounded analytic functions on domains of infinite connectivity, Trans. Amer. Math. Soc. 144 (1969), 241-270.

Department of Mathematics

Daido Institute of Technology

Daido, Minami, Nagoya 457 , Japan 